\title{
Direct Measurement of Medical Linear Accelerator Electron Beam Width at Scattering Foil Position
}

\author{
Tomohiro Shimozato1*, Yuichi Aoyama² \\ ${ }^{1}$ Department of Radiological Technology, School of Health Science, Gifu University of Medical Science, Gifu, Japan \\ ${ }^{2}$ Department of Radiotherapy, Kobe University Hospital, Kobe, Japan \\ Email: *shimo-p@umin.ac.jp
}

How to cite this paper: Shimozato, T. and Aoyama, Y. (2017) Direct Measurement of Medical Linear Accelerator Electron Beam Width at Scattering Foil Position. International Journal of Medical Physics, Clinical Engineering and Radiation Oncology, 6, 73-79.

https://doi.org/10.4236/ijmpcero.2017.61007

Received: January 20, 2017

Accepted: February 21, 2017

Published: February 24, 2017

Copyright () 2017 by authors and Scientific Research Publishing Inc. This work is licensed under the Creative Commons Attribution International License (CC BY 4.0).

http://creativecommons.org/licenses/by/4.0/

\begin{abstract}
Purpose: The aim of this study was to develop a method for the direct measurement of electron beam width and distribution at the scattering foil on the carrousel in a medical linear accelerator gantry head, which differs from an existing indirect method for measuring the focal spot size using a camera or metallic slit located outside the gantry head. Methods: The electron beam emitted by the linear accelerator was used to irradiate radiochromic film mounted on the scattering foil on the carrousel, which was not used for clinical treatment. The electron beam width at the scattering foil position was then evaluated using the full width at half maximum of the Gaussian distribution approximated from each one-dimensional distribution of the irradiated radiochromic film. Results: The electron beam width at the scattering foil position was found to be 3.1 to $6.4 \mathrm{~mm}$ in the crossline direction and 2.8 to $5.5 \mathrm{~mm}$ in the inline direction with electron energy of 4 to $16 \mathrm{MeV}$. The two-dimensional distribution of the electron beam was therefore elliptical or distorted in shape, not circular. Conclusions: Direct measurement of the electron beam width at the scattering foil in the carrousel of a medical linear accelerator is possible, though the use of lower sensitivity film in addition to indirect methods is expected to bring about better results. However, as this method does not allow for direct measurement of the incident angle of the accelerated electron beam, further improvements and refinements are still needed.
\end{abstract}

\section{Keywords}

Medical Linear Accelerator, Electron Beam Distribution, Radiochromic Film, Scattering Foil

\section{Introduction}

The radiation beam of medical linear accelerators (LINACs) used clinically for 
external radiotherapy must be carefully managed and maintained. This involves periodically checking the incident position and angle of the beam axis and the consistency of the beam profile, a method that has been described by Lutz et al. [1] and Karzmark [2]. In previous research into X-ray irradiation used for external radiation therapy, the focal spot size of the electron beam incident on a target was measured using indirect methods involving a spot camera [3] or metallic thin slit [4] [5] [6] located outside the irradiation gantry head. This approach, however, makes it difficult to calculate the distribution of the accelerated electron beam before reaching the X-ray target, as the spread of the focal spot through this target can vary if it is not manufactured uniformly. In the case of the medical LINAC manufactured by Varian Medical Systems, the fact that the target is located in the bending magnet under vacuum makes it particularly difficult to directly measure the focal spot size by inserting a detector. This has created a need for a method to measure the width of the electron beam using detectors located at the exit of the bending magnet. In normal X-ray mode, the target is automatically loaded in the primary collimator. This means that the electron beam is absorbed and scattered by the metallic target, which increases the spread of its distribution after the target.

The focal spot size of an electron beam with a target that is not charged in a primary collimator was measured by Sawkey and Faddegon [7] using the spot camera method proposed by Lutz et al. [3]. They found that a LINAC without a monitor chamber, primary foil, or secondary foil operating in electron mode with a $100-\mathrm{cm}$ source to surface distance (SSD) had a focal spot size that was not scattered. According to Huang et al. [8], it is difficult to accurately reflect the spread of the electron beam as a parameter of Monte Carlo simulation, as it is elliptical or distorted rather than circular. With regard to the electron mode, the only scattering object before the scattering foil (SF) is a $0.00254 \mathrm{~cm}$-thick [9] beryllium $(\mathrm{Be})$ window in the beam duct exit. The aim of this study is therefore not to measure the focal spot diameter at the X-ray target position, but rather to develop a technique to directly determine the electron beam width and distribution at the SF position via irradiation of the film on the carrousel.

\section{Materials and Methods}

All measurements were conducted on a medical LINAC (Clinac 2100CD, Varian Medical Systems, Palo Alto, CA) that is not used for clinical treatment and with the SF bracket removed from the carrousel. This was operated in "service mode" with several hardware and software interlocks overridden, mainly to allow for beam delivery and control over the dose by servo. The steering switch was also turned off when adjusting the focal spot position.

A radiochromic film (GafChromic film MD-55, Ashland Inc., Bridgewater, NJ, USA) was inserted into the SF bracket mounted on the carrousel instead of the tantalum foil normally used with an electron beam energy of $4 \mathrm{MeV}$. We used because MD-55 is less sensitive than GafChromic film EBT2 or EBT3. Electron beams with energies of $4,6,9,12$, and $16 \mathrm{MeV}$ were then emitted from the 
LINAC, resulting in an irradiation dose of 1 monitor unit (MU) at a dose rate of $100 \mathrm{MU} / \mathrm{min}$. The positions of the SFs and flattening filters (FFs) on the carrousel [10] are shown in Figure 1.

These positions were determined based on the electron or photon energy selected by the control panel (4-D console) of the LINAC. Figure 2 shows the geometrical position [9] of the bending magnet, Be window, and carrousel. Figure 3 shows the SF bracket with radiochromic film mounted on the hole on the carrousel to ensure that the angle between the beam exit and gantry head of the LINAC is $0^{\circ}$. This also shows the film insertion position described in Figure 2, for which several lead blocks normally used for leakage radiation shielding were removed.

Since the SF bracket could not be installed directly on the beam axis (the "beam irradiation position" in Figure 2) relative to the geometrical structure, the film was mounted on the "film insertion position" and moved by an air

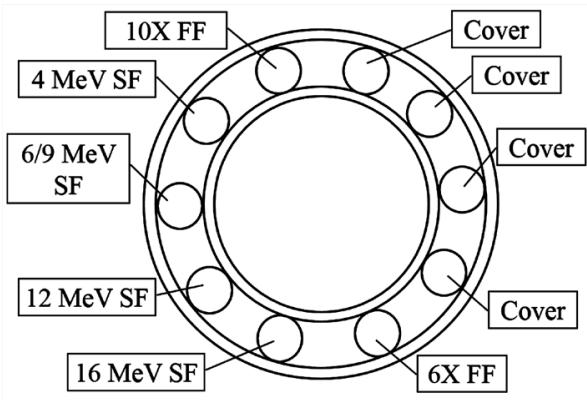

Figure 1. Position of scattering foils for different energies [10] and flattening filters on carousel of medical linear accelerator.

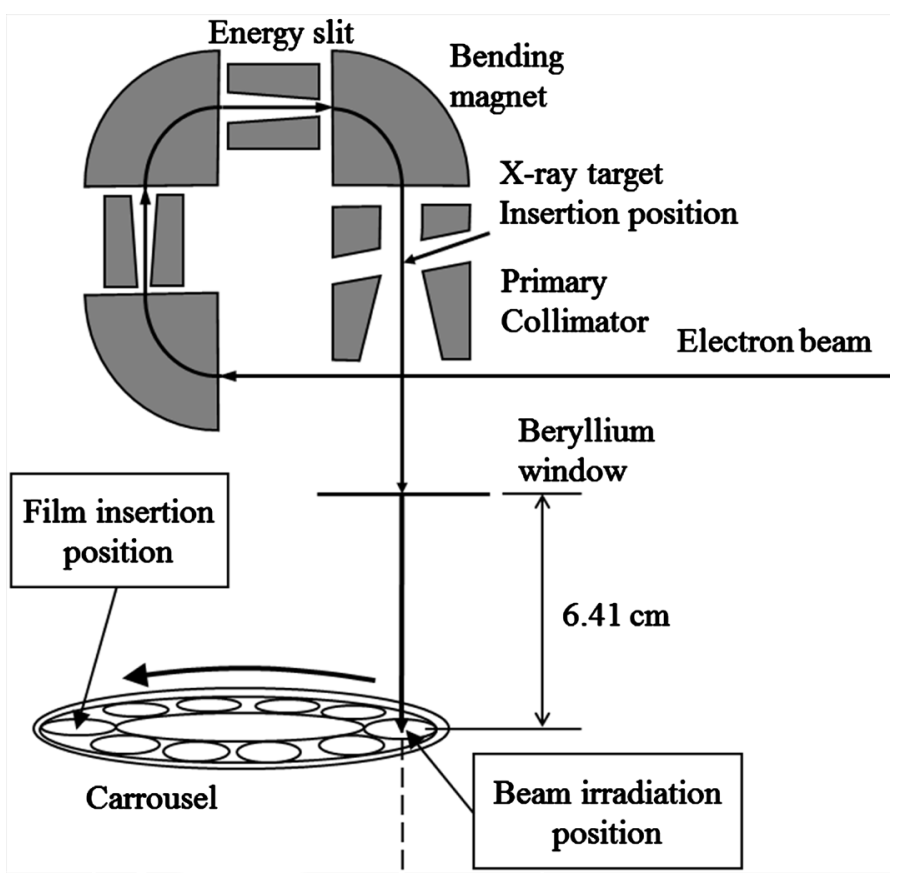

Figure 2. Film insertion position and beam irradiation position on carrousel in medical linear accelerator gantry head [9]. 
compressor driving the carrousel to the "beam irradiation position." When electron beam was irradiated to the "beam irradiation position", the lead blocks were returned to the original position. The irradiated film was digitized by a flatbed scanner (Epson $10000 \mathrm{G}$ ) $24 \mathrm{~h}$ after irradiation using a spatial resolution of 300 dots per inch. The resulting two-dimensional distribution of optical density was saved in a text-style format used by film analysis software DD-system Ver. 9.4 (R-TECH Inc.). Crossline and inline direction distributions from this two-dimensional text data were extracted and evaluated. The full width at half maximum (FWHM) of similar Gaussian distributions with measured film distributions was then evaluated to find the electron beam width.

\section{Results and Discussions}

The pictures in Figure 4 of the irradiated radiochromic film show that the two-dimensional distribution of optical density was elliptical or distorted, not circular. The shape of the two-dimensional distribution is different in each energy. It is difficult to consider the impact of the flatbed scanner. The diameter of the dark spot also decreased with increasing beam energy. Although the electron beam accelerated by the LINAC is considered to have a very narrow focus, it would appear that the accelerated incident electron beam is scattered by its interaction with the very thin Be window at the bending magnet exit and the air

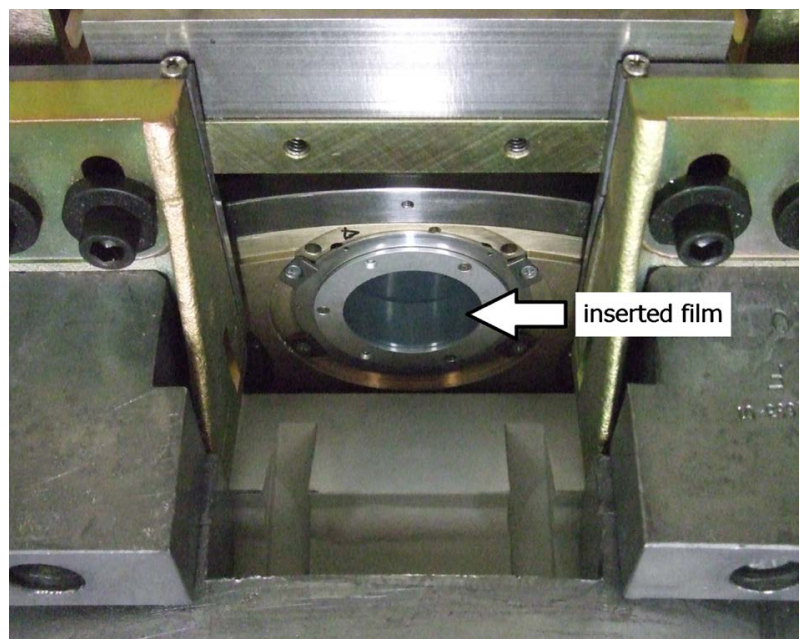

Figure 3. Picture of scattering foil bracket with MD-55 radiochromic film at "film insertion position" shown in Figure 2.
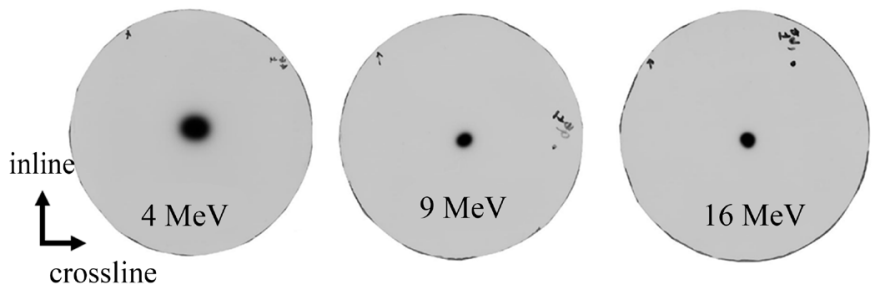

Figure 4. Two-dimensional optical density distribution of radiochromic film irradiated by (a) 4, (b) 9, and (c) $16 \mathrm{MeV}$ electron beams. 
slab between this Be window and the measurement position at the SF. These electron beam widths were larger than the focal spot size observed in previous papers [8] due to the distance of $12.5 \mathrm{~cm}$ from the position of the target in $\mathrm{x}$-ray mode to the measurement point.

Figure 5 shows the Gaussian distribution and one-dimensional distribution of
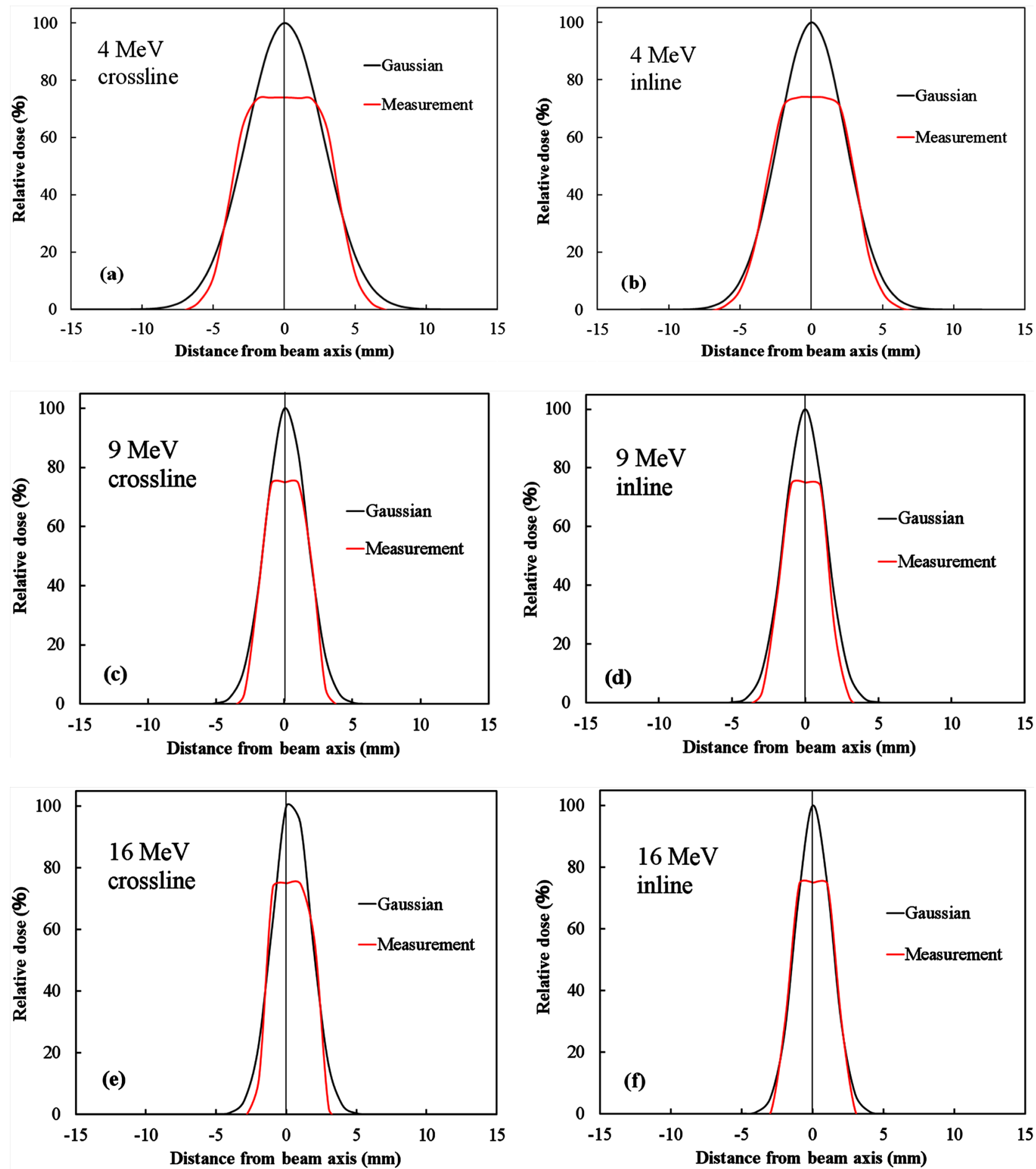

Figure 5. One-dimensional distribution of irradiated film (red line) and similar Gaussian distribution (black line) in the (a) (c) (e) crossline and (b) (d) (f) inline direction for beam energies of (a) (b) 4, (c) (d) 9 and (e) (f) $16 \mathrm{MeV}$. 
the net optical density of the films irradiated using different electron beam energies. Note that the density in the region around the beam axis of the one-dimensional distribution is lower than the Gaussian distribution, which indicates that the sensitivity threshold of the film was exceeded in this high-dose region. The fact that the absorbed dose of $1 \mathrm{MU}$ exceeded the sensitivity threshold also meant that it was not possible to construct a calibration curve for converting net optical density to absorbed dose. Thus, although the radiochromic MD-55 film used in this study has a very low sensitivity for medical measurement, and has been used for patient QA in stereotactic radiosurgery, an even lower sensitivity film (e.g., industrial-grade film) should make it possible to convert the net optical density to absorbed dose.

Table 1 presents the FWHM values for the Gaussian distribution obtained from the one-dimensional distribution in the crossline and inline direction of the irradiated radiochromic film. The maximum FWHM was $6.4 \mathrm{~mm}$ in the crossline direction and $5.5 \mathrm{~mm}$ in the inline direction at $4 \mathrm{MeV}$, whereas the minimum FWHM was $3.1 \mathrm{~mm}$ in the crossline direction and $2.8 \mathrm{~mm}$ in the inline direction at $12 \mathrm{MeV}$. The diameter of the electron beam spread reduced as the electron beam energy increased; however, the distribution of the beam remained as an incomplete circle similar to that identified by Jaffray et al. [4], as there was a consistent difference between the widths in the crossline and inline directions. Since the distance [9] between the X-ray target and measurement position on the carrousel was very small $(12.5 \mathrm{~cm})$ when compared to normal treatment distances of $100 \mathrm{~cm}$, it is unsurprising that the irradiation dose exceeded the sensitivity threshold of the film. Further analysis of the high-dose region using a lower sensitivity film would allow the Gaussian distributions to be compared, making it possible to determine the position of the incident electron beam rather than just its width and distribution. The method presented in this study also requires removing the SF bracket, as the position of the carrousel was determined by the electron energy selected. As such, if the position of the carrousel determined by electron energy could select the position of the cover, as shown in Figure 1, then the position and width of the incident electron beam could conceivably be periodically measured during clinical treatment.

\section{Conclusion}

This study has proposed a useful method for directly measuring the width of an electron beam at the scattering foil position on its carrousel for clinical LINACs.

Table 1. FWHM of the Gaussian distribution obtained from the one-dimensional distribution of irradiated film.

\begin{tabular}{cccccc}
\hline FWHM $(\mathrm{mm})$ & \multicolumn{5}{c}{ Electron Energy } \\
\hline Direction & $4 \mathrm{MeV}$ & $6 \mathrm{MeV}$ & $9 \mathrm{MeV}$ & $12 \mathrm{MeV}$ & $16 \mathrm{MeV}$ \\
\hline Crossline & 6.4 & 4.5 & 3.5 & 3.1 & 3.2 \\
Inline & 5.5 & 3.9 & 3.3 & 2.8 & 2.9 \\
\hline
\end{tabular}


Direct measurement of the electron beam width at the scattering foil in the carrousel of a medical linear accelerator is possible. In this case, the position of the carrousel was determined by the electron energy, but if it could be selected freely, then it should be possible to measure the electron beam distribution at the SF position using radiochromic film at one of the cover positions on the carrousel during clinical treatment. However, as this method does not allow for direct measurement of the incident angle of the accelerated electron beam, further improvements and refinements are still needed.

\section{Conflict of Interest}

The authors have no conflict of interest to report.

\section{References}

[1] Lutz, W.R., Romald, R.D. and Bjarngard, B.E. (1981) Beam Alignment Tests for Therapy Accelerators. International Journal of Radiation Oncology ${ }^{\star}$ Biology ${ }^{\star}$ Physics, 7, 1727-1731. https://doi.org/10.1016/0360-3016(81)90201-7

[2] Karzmark, C.J. (1984) Advance in Linear Accelerator Design for Radiotherapy. Medical Physics, 11, 105-128. https://doi.org/10.1118/1.595617

[3] Lutz, W.R., Maleki, N. and Bjarngard, B.E. (1988) Evaluation of a Beam-Spot Camera for Megavoltage X-Rays. Medical Physics, 15, 614-617.

https://doi.org/10.1118/1.596214

[4] Jaffray, D.A., Battista, J.J., Fenster, A. and Munro, P. (1993) X-Ray Sources of Medical Linear Accelerators: Focal and Extra-Focal Radiation. Medical Physics, 20, 1417-1427. https://doi.org/10.1118/1.597106

[5] Sham, E., Seuntjens, J., Devic, S. and Podgorsak, E.B. (2008) Influence of Focal Spot on Characteristics of Very Small Diameter Radiosurgical Beams. Medical Physics, 35, 3317-3330. https://doi.org/10.1118/1.2936335

[6] Anai, S., Arimura, H., Nakamura, K., Araki, F., Matsuki, T., Yoshikawa, H., Yoshidome, S., Shioyama, Y., Honda, H. and Ikeda, N. (2011) Estimation of Focal and Extra-Focal Radiation Profiles Based on Gaussian Modeling in Medical in Medical Linear Accelerators. Radiological Physics and Technology, 4, 173-179. https://doi.org/10.1007/s12194-011-0118-1

[7] Sawkey, D.L. and Faddegon, B.A. (2009) Determination of Electron Energy, Spectral Width, and Beam Divergence at the Exit Window for Clinical Megavoltage X-Ray Beams. Medical Physics, 36, 698-707. https://doi.org/10.1118/1.3070547

[8] Huang, V.W., Senuntjens, J., Devic, S. and Verhaegen, F. (2005) Experimental Determination of Electron Source Parameters for Accurate Monte Carlo Calculation of Large Electron Therapy. Physics in Medicine and Biology, 50, 779-786. https://doi.org/10.1088/0031-9155/50/5/004

[9] Varian Medical Systems (2011) Monte Carlo Data Package High Energy Accelerator DWG No. 100040466-02. Rev.2.

[10] Varian Medical Systems (2010) High Energy Systems (Clinac iX, Trilogy \& Clinac Family) Data Book 100021350-02 Section 5. 
Submit or recommend next manuscript to SCIRP and we will provide best service for you:

Accepting pre-submission inquiries through Email, Facebook, LinkedIn, Twitter, etc. A wide selection of journals (inclusive of 9 subjects, more than 200 journals)

Providing 24-hour high-quality service

User-friendly online submission system

Fair and swift peer-review system

Efficient typesetting and proofreading procedure

Display of the result of downloads and visits, as well as the number of cited articles Maximum dissemination of your research work

Submit your manuscript at: http://papersubmission.scirp.org/

Or contact ijmpcero@scirp.org 